\title{
Taller de sensibilización ante la violencia de pareja promovida por los mitos del amor romántico
}

\author{
The Process of Raising Awareness of Partner Violence Fostered by Romantic Love Myths \\ Mitzi Abigail Chora López, Iris Xóchitl Galicia Moyeda, Brenda Giselle Guerrero García \\ Facultad de Estudios Superiores Iztacala
}

\begin{abstract}
Resumen
Los mitos del amor romántico están presentes en el estudiantado universitario y pueden conducir a comportamientos violentos en sus relaciones de pareja. Por lo anterior, se realizó un taller con 11 alumnos universitarios de $6 .{ }^{\circ}$ semestre de psicología con el fin de sensibilizarlos hacia comportamientos realizados en las relaciones de pareja que son promovidos por los medios de comunicación y que reproducen los mitos del amor romántico. Mediante un cuestionario, se analizaron sus respuestas ante algunos comportamientos violentos en las parejas antes y después del taller. Posterior al taller, los participantes rechazaron esos comportamientos en su persona y en sus relaciones de pareja, argumentando que respetan a otras personas que aceptan los mitos románticos. En la discusión, se argumenta que este tipo de respuesta es considerado como un proceso de sensibilización incipiente.
\end{abstract}

Palabras clave: estudiantado universitario, violencia de pareja, mitos del amor romántico, sensibilización, interacción social.

Mitzi Abigail Chora López. Facultad de Estudios Superiores Iztacala, UNAM.

Iris Xóchitl Galicia Moyeda. Facultad de Estudios Superiores Iztacala, UNAM.

https://orcid.org/0000-0002-8287-710X

Brenda Giselle Guerrero García. Facultad de Estudios Superiores Iztacala, UNAM.

Autor para correspondencia: iris@unam.mx 


\begin{abstract}
University students sustain romantic love myths that could lead to violent behavior in their love relationships. Therefore, a workshop was carried out with eleven university students majoring in Psychology to raise awareness on behaviors in love relationships that might be fostered by the perpetuation of romantic love myths in media. Participant reactions to this type of behavior were analyzed by means of a questionnaire. Following the workshop, they reported that they did not display this behavior in their love relationships and mentioned they respect other people who accept romantic love myths. The response is discussed as a process of emerging awareness.
\end{abstract}

Keywords: university students, partner violence, romantic love myths, raising awareness, social interactions.

DOI https://doi.org/10.36793/psicumex.v11i2.369

Recibido 26 - Agosto - 2020

Aceptado 24 - Agosto - 2021

Publicado 25 - Septiembre - 2021 


\section{Introducción}

Dentro de las relaciones que el ser humano establece, tienen lugar y relevancia las relaciones de pareja, que hacen referencia a la vinculación afectiva con una persona fuera del núcleo familiar con quien se decide de manera consciente compartir factores que socialmente se consideran importantes.

En las relaciones de pareja se presencian situaciones conflictivas debido al incumplimiento de expectativas e ideales fundamentados en los estereotipos de papeles de género que, de acuerdo con Samaniego y Freixas (2010), refieren a comportamientos socialmente esperados de acuerdo con el género al que se pertenece. Las y los jóvenes al encontrarse en una etapa de constante cambio y búsqueda de identidad ponen atención a dichos ideales, lo que puede conducir gradualmente a episodios violentos en las relaciones que establecen. Según Linares y Sologuren (2012), los jóvenes universitarios le atribuyen a la relación de pareja un significado que implica el reconocimiento de la identidad y la necesidad de compartir sentimientos, esto propicia la realización de conductas y actitudes para fortalecer el vínculo amoroso y excluir a los demás de su relación para construir espacios de vida personal con su pareja, lo que puede llevar a comportamientos violentos.

Algunos investigadores consideran al nivel educativo como un factor desencadenador diferencial de violencia de pareja. Por ejemplo, Rivera-Rivera et al. (2006) compararon el índice de violencia de pareja en estudiantes de secundaria y bachillerato-universidad. Los estudiantes de secundaria mostraban índices menores de violencia física y psicológica a diferencia de los del nivel de bachillerato-universidad donde los puntajes aumentaban ligeramente. Los datos anteriores parecen sugerir que la violencia de pareja en estudiantes depende del grado académico que cursen. Aunque aceptar tal sugerencia resultaría inadecuado debido a que hasta el momento no se tiene identificado un patrón específico. Rojas (2013) menciona que la baja frecuencia de la violencia psicológica en las relaciones de pareja entre adolescentes se debe a que suelen identificar más 
fácilmente las agresiones físicas o sexuales antes que las psicológicas. No obstante, la violencia psicológica es la más frecuente. Diversos estudios confirman esta tendencia tanto en la población general como en la comunidad universitaria (CONAPO, 2014; Sánchez y Solís, 2009; Castro y Casique, 2010; Rodríguez-Franco et al., 2010; Rosales, Flórez y Fernández, 2017; GarcíaCarpintero, Rodríguez-Santero y Porcel-Gálvez, 2017).

Al respecto, Olvera, Arias y Amador (2012) trabajaron en un centro universitario del Estado de México con estudiantado femenino. Ellos reportan que el $31 \%$ de las participantes identificaron tres tipos de violencia (psicológica, sexual y física) en sus relaciones de pareja. Del total de las participantes, el $92 \%$ reconoció indicios de violencia psicológica, la cual con frecuencia estaba acompañada de otro tipo de violencia, específicamente, el 34\% de las mujeres universitarias manifestaron vivir episodios de violencia psicológica combinada con alguno de los otros tipos. Otra investigación con estudiantado universitario (Galicia, Robles y Sánchez, 2020) valoró la violencia psicológica en la pareja y se advirtió que la conducta de control en comparación con la de chantaje está más presente entre el estudiantado y que las mujeres la identifican en mayor medida que los hombres. Estos datos coinciden en alguna medida con lo encontrado por Corral y Zamudio (2010) y por Andrade, Arana y Alvarado (2017) quienes evidencian que son las mujeres quienes más sufren la violencia psicológica entre la población universitaria

Los resultados de estas investigaciones no respaldan la de idea de que los estudiantes universitarios, por estar en un nivel educativo superior, son menos susceptibles de realizar o padecer conductas violentas. Esos resultados pueden explicarse considerando el proceso de socialización al que los individuos se encuentran expuestos y que está presente en las conductas expresadas, incluso por los universitarios, en diversas situaciones. Como resultado de este proceso de socialización, las personas aprenden y se apropian de pautas de comportamiento de su medio social que forman el concepto de amor romántico, asociándolo a los mitos compartidos 
culturalmente (Ferrer y Bosch, 2013). Con la influencia de agentes socializadores se hace una diferenciación de género para crear en las personas estilos cognitivos y de conducta que son dirigidos por normas estereotípicas, asociando de esta manera diferencias sociales entre los hombres y las mujeres, colocando a los hombres y la masculinidad en un rol de poder, mientras que a la mujer se le considera en un rol femenino de dependencia y obediencia. Con esto se incorporan elementos que dan pie a comportamientos violentos. Además, estos autores señalan que en las relaciones de pareja se retoma el concepto de amor romántico como el amor que implica intimidad y pasión sin compromiso, y que es transmitido por el proceso de socialización, creando de esta forma altas expectativas que generan consecuencias personales y sociales de insatisfacción, frustración y sanción social si no son cumplidas.

Cabe señalar que el amor romántico deriva del mito andrógino de Platón, el cual narra la historia de seres que tenían las características de ambos sexos, por lo tanto, eran duales y completos en sí mismos. Cuando intentaron invadir el monte Olimpo, Zeus les lanzó un rayo que hizo que se dividieran en dos, en consecuencia, estaban condenados y castigados a buscar a su otra mitad (Nadal, 2003). Este mito fue una de las bases para justificar el amor romántico occidental, por otra parte, también es considerado heredero del amor cortés, del amor burgués y del victoriano por consolidar la dependencia entre hombres y mujeres, argumentando una supuesta necesidad de complementación (Pascual, 2016). Otro de los mitos del amor romántico se basa en asociar la consecución del amor con la felicidad y la plenitud, haciendo que la búsqueda de una pareja sea una meta fundamental en la vida. Las diferentes ideas de amor romántico que se encuentran en la sociedad son, por ejemplo: la idea de la entrega total hacia el otro, hacer del otro lo único y fundamental de la existencia; consagrarse al bienestar del otro, depender del otro y adaptarse a él, postergando lo propio; idealizar a la otra persona, negando la existencia de algún defecto; perdonar 
y justificar todo en nombre del amor; vivir experiencias muy intensas de felicidad o de sufrimiento y pensar que es imposible volver a sentir esa intensidad.

En relación con lo anterior, Pascual (2016) menciona que la ideología del amor romántico ha servido a los distintos poderes para perpetuar un sistema social que fomenta la desigualdad entre géneros y desfavorece en gran medida al femenino. Este tipo de amor es tema central de muchos productos culturales, generando discursos que impactan en la construcción de identidad de las personas, sobre todo en los jóvenes, de ahí que los medios de comunicación se conviertan en aliados que difunden las ideas del amor romántico.

Ahora bien, la asimilación de los mitos y creencias sobre el amor romántico puede generar tolerancia a la violencia en la relación debido a ideas como el amor lo puede todo o es normal sufrir por amor. Además, adoptar ese modelo de amor y sus mitos dificulta la reacción ante una situación de violencia como lo mencionan Ferrer y Bosch (2013), pues creencias como "el amor es lo que da sentido a la vida", "el renunciar al amor es un fracaso", "el amor todo lo puede" o "la violencia y el amor son compatibles" conllevan una serie de ideas creadas tanto para justificar y aceptar comportamientos violentos como para retrasar una ruptura amorosa y la búsqueda de ayuda. Según Deza (2012), la percepción positiva del amor romántico es uno de los factores con más relevancia para la permanencia en una relación violenta de pareja.

Los medios de comunicación juegan un papel muy importante en el proceso de socialización de tales mitos desde la infancia hasta la juventud. Tienen un fuerte impacto en la sociedad, sobre todo en la configuración de valores, creencias y actitudes (Cabero y Romero, 2011). Los medios influyen en la idealización de las relaciones de pareja porque sus elementos apelan a la emoción; estas ficciones audiovisuales no se enfocan en la razón sino en la creación y transmisión de estructuras imaginarias y sentimentales (Pascual, 2016). 
Atendiendo lo anterior, si se desea disminuir la violencia de pareja en una comunidad dada, se propone que una manera de intervenir es encauzar a sus integrantes, tanto a hombres como mujeres, hacia un proceso de sensibilización hacia las manifestaciones de esa violencia disfrazadas por los patrones del amor romántico prevalecientes en diversos productos de los medios de comunicación. Se habla de sensibilización pues esta implica que el individuo alcance la capacidad de valorar algo o de responder emocionalmente ante ello. Así pues, una acción para disminuir la violencia de pareja consistiría en realizar un proceso de sensibilización hacia ella. Sensibilizar permite un acercamiento afectivo y una implicación en los problemas a través del ejercicio de procesos de reflexión y autorreflexión para favorecer la toma de conciencia (Virgilí, 2014). Esta investigadora menciona que, para promover una sensibilización, primero, debe reconocerse la existencia de una desigualdad en el plano personal, y después, reconocer que es una realidad compartida por un colectivo, que no es sólo de orden particular, para que así haya una implicación en la transformación personal y en el cambio social.

La comunidad de estudiantes universitarios ha mostrado aceptar los mitos del amor romántico (Galicia, Robles, Sánchez y Núñez, 2019) y comportarse conforme a ellos. Por lo que es deseable que en esta comunidad se realicen actividades que faciliten la comprensión de que ciertos comportamientos en las relaciones de pareja son una forma de manifestar los mitos del amor romántico propiciados por los medios de comunicación y que tienen una alta probabilidad de conducir a comportamientos violentos en la pareja. Por lo cual, el objetivo del presente reporte es mostrar los efectos de un taller que tuvo como propósito sensibilizar a los estudiantes de psicología de una universidad pública sobre algunos mitos del amor romántico presentes en los medios de comunicación y como estos pueden generar comportamientos asociados a la violencia en la pareja. 


\section{Método}

\section{Participantes}

Se invitó a participar en un taller sobre relaciones de pareja a alumnos que cursaban el sexto semestre de la licenciatura de psicología de una facultad ubicada en el Estado de México, en el entendido de que ya habían obtenido conocimientos de los temas de violencia en algunas de las asignaturas cursadas en semestres anteriores.

De esta forma, el criterio de inclusión fue que los participantes estuvieran cursando el sexto semestre y además refirieran haber revisado contenidos sobre violencia en algunas de las asignaturas cursadas hasta ese momento. Fueron excluidos aquellos estudiantes que no cumplieran dicho criterio. Los estudiantes que mostraron interés en participar fueron informados de que se realizarían dos evaluaciones, al inicio y final del taller, y de que los resultados obtenidos serían tratados con confidencialidad y no se revelarían datos con los cuales se les pudiera identificar. También se les notificó que en el momento que desearan salir del estudio podrían hacerlo sin ninguna consecuencia. Una vez aceptada esta situación, los participantes firmaron un consentimiento.

En un inicio, los participantes del taller fueron once estudiantes, ocho mujeres y tres hombres, de entre 20 y 24 años, quienes asistieron de manera irregular al taller. De ellos, solamente dos mujeres y un hombre asistieron a la última sesión (que coincidió con el último día del semestre escolar) donde se aplicó una segunda evaluación. Es por ello que los datos reportados en la pre y post evaluación son únicamente de esos tres participantes. Uno de ellos era hombre, identificado con la letra $\mathrm{H}$ y los restantes, dos mujeres, identificadas con la letra M.

\section{Instrumento}

Se empleó un cuestionario, elaborado exprofeso, con quince situaciones divididas en cinco constructos que hacen referencia a comportamientos relacionados con los mitos del amor 
romántico. Los mitos elegidos se retomaron de Luzón et al. (2011), se identifican con el número que le asignaron dichos autores y se enlistan a continuación:

Mito 6: representa la creencia en que el amor verdadero lo perdona y/o justifica todo.

Mito 10: refleja la creencia en que solo hay un amor verdadero en la vida.

Mito 12: alude a la conversión del amor de pareja en lo fundamental de la existencia.

Mito 14: la entrega total, que se refiere a la idea de "fusión con el otro", al olvido de la propia vida, a la dependencia de la otra persona y adaptación a ella, postergando lo propio. Esta creencia está íntimamente relacionada con la concepción del amor como sacrificio por la otra persona.

Estos mitos se reflejan en ideas prevalecientes sobre las relaciones amorosas y fueron expresados en enunciados que aludían a esas ideas; para efectos de este trabajo fueron identificados como constructos. Por ejemplo, el mito 6, que representa la creencia en que el amor verdadero lo perdona y/o justifica todo, se identifica en este trabajo como constructo con la frase "perdonar y justificar todo en nombre del amor". Para cada constructo se elaboraron enunciados que reflejaban situaciones comunes en una relación de pareja, los cuales se sometieron a la revisión de tres profesores considerados expertos en el tema de violencia. De tal revisión se aceptaron quince enunciados, que fueron los que conformaron el instrumento empleado.

En la tabla 1 se presentan esas situaciones, agrupadas junto al constructo que ejemplificaban. En el cuestionario que se les proporcionó a los participantes, se presentaron dichas situaciones en el mismo orden de la tabla, sin identificar el constructo al que pertenecían. Las instrucciones fueron: “A continuación, te encontrarás una serie de situaciones, responde marcando con una X sí o no según consideres adecuadas las siguientes conductas en una relación de pareja y a continuación justifica tu respuesta”. 


\section{Tabla 1}

Situaciones empleadas en el cuestionario agrupadas por constructo

Constructos a evaluar

a) Depender del otro $y$ adaptarse a él, postergando lo propio (Corresponde al mito 14: la entrega total)

\section{Reactivos}

1. José lleva meses esperando a que se estrene una película que le llama mucho la atención, pero no está en disposición de ir si no va con su pareja.

2. Sergio cambió de turno en su trabajo porque se le presentaron mejores oportunidades al hacerlo. María (su novia), a pesar de que tiene estabilidad en su trabajo y la posibilidad de tener un ascenso, decide cambiar de turno para poder estar más tiempo con Sergio.

3. Dany habla con su pareja todas las noches y no puede conciliar el sueño hasta recibir un mensaje o una llamada de él.

b) Prestar atención y vigilar 4. Cada vez que cumplen meses de ser novios, la pareja de Martha le regala cualquier señal o signo de altibajos en el amor o en un detalle (un chocolate, una carta, una rosa, etc.) pero este mes se le ha olvidado y Martha está muy enojada.

interés del otro. Es una forma de expresar el mito 12 , que alude a la 5. La pareja de Jaime se está comunicando con él por WhatsApp y le dice que irá a dormirse. A pesar de que ya se despidió de él, Jaime entra al chat constantemente para ver si ella sigue "en línea".

conversión del amor de 6. La pareja de Juan todo el tiempo está al pendiente de a qué hora y en qué pareja en lo fundamental de la existencia. lugares se encuentra Juan. Cuando él le dice estar en casa su pareja marca para asegurarse que sea verdad. 


\section{Tabla 1 (continuación)}

Muestra las situaciones empleadas en el cuestionario, agrupadas por constructo

\section{Constructos a evaluar}

Reactivos c) Sentir que nada vale más que esa relación (Corresponde al mito 10: refleja la creencia en que solo hay un amor verdadero en la vida) d) Perdonar y justificar todo en nombre del amor (corresponde al mito 6 que representa la creencia en que el amor verdadero lo perdona y/o justifica todo);
7. Antonio tiene un trabajo escolar final muy importante para concluir el semestre, pero en lugar de hacerlo prefiere salir a pasear con su novia porque ella es más importante.

8. Alfredo salía mucho con sus amigos de fiesta, a comer o a cualquier lugar, pero desde que inició la relación con su pareja, poco a poco ha dejado de salir e incluso de hablarle algunos amigos porque a él le interesa mucho su relación. 9. Carlos tiene el sueño de participar en un concurso de robótica que es en otro estado del país; ha llegado casi la fecha y ha decidido no inscribirse porque el concurso será un fin de semana. Carlos ve a su novia los fines de semana, como ella es muy importante para Carlos, ha decidido no participar.

10. Francis se enteró de que su pareja le fue infiel. Del impacto no se repone y cae en depresión porque la amó mucho. Está pensando que la vida no es la misma sin ella y está considerando seriamente en otorgarle el perdón.

11. Mariana está muy enamorada de su novio, aunque a veces pelean mucho e incluso él la ha llegado a jalonear y gritarle en público. Para justificarlo, Mariana siempre dice a sus amigos este lema" Soy la más feliz a su lado y la más infeliz sin él"

12. Joselo no deja que su novia use vestidos, faldas, blusas y accesorios bonitos por temor a que lo engañe, pero ella lo justifica diciendo que lo hace por su bien y seguridad.

\begin{tabular}{|c|c|}
\hline & 13. Andy lleva cinco años en su relación y a pesar de que a estas alturas hay \\
\hline volver a amar con esa & más momentos negativos que positivos, Andy cree que sólo es una mala racha \\
\hline intensidad & y que pronto lo van a superar y volverán a amarse intensamente. \\
\hline es una forma de & 14. René considera que durante su relación le ha contado muchas cosas a su \\
\hline anifestar el mito 10 , que & oareja y que ella lo conoce perfectamente, por ende, cree que no va \\
\hline fleja la creencia en que & posible llegar a confiar en alguien más de esa manera. \\
\hline lo hay un amor & 15. Samy ha hecho muchas cosas por su pareja, de las que incluso no se creía \\
\hline rdadero en la vida; & $\begin{array}{l}\text { capaz. Piensa que eso, sólo podría hacerlo por el amor de su vida porque } \\
\text { considera que su actual pareja es esa persona especial. }\end{array}$ \\
\hline
\end{tabular}




\section{Materiales}

Se emplearon canciones, videos, segmentos de películas, anuncios y artículos publicados en revistas y redes sociales de circulación entre el estudiantado universitario.

\section{Procedimiento}

Para llevar a cabo el taller, se solicitó la autorización y un espacio a la Jefatura de la Carrera de Psicología. Se aprobó la actividad y el uso de un aula durante noventa minutos una vez a la semana por un periodo de ocho semanas durante el horario matutino. Durante las sesiones, los asientos del aula se acomodaron formando una media luna para que las y los asistentes al taller y las talleristas (autoras de este reporte) tuvieran una mejor interacción.

\section{Desarrollo del taller}

En todas las sesiones se empleó una metodología participativa basada en un aprendizaje significativo y giró en torno a la reflexión por parte de los y las participantes; para ello, se tomaron en cuenta los conocimientos sobre violencia de pareja que poseían previamente y se relacionaron con la información proporcionada en las sesiones del taller referente a los mitos del amor romántico. Siempre se promovió un proceso de reflexión individual y colectiva de los y las participantes, y que ellos compartieran sus sentimientos, opiniones, experiencias y reflexionaran sobre éstas y los conocimientos relacionados con el tema de violencia de pareja. Las actividades realizadas implicaban identificar comportamientos incluidos en los mitos mencionados anteriormente y reflexionar sobre su relación con la violencia de pareja. En cada sesión se trabajaba cada uno de los constructos enlistados y descritos en la sección de instrumentos. La primera y última sesiones consistieron en introducir y concluir el taller, respectivamente, además de aplicar el test.

En la primera sesión tras recoger el cuestionario, se dio una plática usando como apoyo una presentación de PowerPoint, donde se abordó el tema del amor romántico y los mitos que hay 
alrededor del mismo. Se hizo énfasis en su inadecuada interpretación en las relaciones afectivas entre los y las jóvenes, que en muchas ocasiones favorece que se desarrollen creencias e imágenes idealizadas de esas relaciones que, en numerosas ocasiones, promueven una tolerancia a comportamientos abusivos y violentos en las relaciones afectivas.

La segunda sesión tuvo como objetivo reflexionar sobre las consecuencias de conductas destinadas a prestar atención y vigilar cualquier señal o signos de altibajos en el amor o interés en el otro, realizando un juego de roles entre las y los participantes. El objetivo de la tercera sesión consistió en reconocer la existencia del mito relativo al perdón inmerso en las canciones que son de su agrado. Para ello, se revisaron y analizaron las letras de canciones románticas de la preferencia de los y las participantes.

En la cuarta sesión, se trabajó sobre la creencia de que sólo hay un amor verdadero y se reflexionó el cómo esta idea podría propiciar violencia en las relaciones de pareja. En esta ocasión se realizó un análisis de la película El diario de una pasión y de otras que los participantes compartieron.

Los objetivos de la quinta sesión fueron identificar y reflexionar sobre las conductas de dependencia hacia el otro y de postergación de lo propio como una muestra de amor. Para ello, se analizó cómo dichas conductas son promovidas por las revistas de moda de circulación entre el estudiantado universitario.

Para la sexta sesión el objetivo fue reflexionar sobre las consecuencias de validar la idea de que nada vale más que la relación de pareja. Para ello, se realizó un collage y, a partir de él, los y las participantes expresaron sus argumentos para abordar y detectar indicadores de abuso en las parejas jóvenes y la importancia de mantener relaciones igualitarias.

En la séptima sesión se realizó un recuento de lo revisado en sesiones anteriores. Para ello, se escucharon y analizaron diferentes temas de canciones, y se solicitó argumentar cómo las 
canciones y otros productos culturales y audiovisuales que se consumen a diario, por ejemplo en radio y/o televisión, reproducen los mitos del amor romántico antes expuestos y cómo estos influyen en el tipo de relaciones afectivas y emocionales que construyen las parejas. También, se mostraron mensajes que predominan en las redes sociales donde se acepta e interioriza la violencia y el uso de la agresividad para mantener una relación afectiva.

En la última sesión, la octava, se dio un agradecimiento a los participantes y se aplicó de nuevo el instrumento a manera de postest.

\section{Resultados}

Únicamente tres participantes realizaron las dos evaluaciones y son los resultados que se presentan. Se identificaron las respuestas que manifestaban desacuerdo y acuerdo en cada uno de los reactivos. Las respuestas que estaban de acuerdo con lo planteado en el reactivo se representan con el número 1 y las que estaban en desacuerdo con el número 2. Lo anterior se presenta en la tabla 4, en donde las filas sombreadas corresponden a las respuestas dadas en la evaluación final o postest

\section{Tabla 2}

Respuestas de acuerdo y en desacuerdo con las situaciones planteadas en cada uno de los reactivos del instrumento para los tres participantes durante el pre y el post test

\begin{tabular}{|c|c|c|c|c|c|c|c|c|c|c|c|c|c|c|c|c|}
\hline & & \multicolumn{15}{|c|}{ Constructos } \\
\hline & & \multicolumn{4}{|c|}{1} & \multicolumn{2}{|l|}{2} & \multicolumn{3}{|c|}{3} & \multicolumn{3}{|c|}{4} & \multicolumn{3}{|c|}{5} \\
\hline & & \multicolumn{15}{|c|}{ Reactivos } \\
\hline Evaluaciones & Participantes & 1 & 2 & 3 & 4 & 5 & 6 & 7 & 8 & 9 & 10 & 11 & 12 & 13 & 14 & 15 \\
\hline Pre & M1 & 2 & 2 & 3 & 2 & 2 & 2 & 2 & 2 & 2 & 2 & 2 & 2 & 2 & 2 & 2 \\
\hline Post & M1 & 1 & 1 & 2 & 2 & 2 & 2 & 1 & 1 & 2 & 2 & 2 & 2 & 1 & 1 & 1 \\
\hline Pre & M8 & 2 & 2 & 2 & 2 & 2 & 2 & 2 & 2 & 2 & 2 & 2 & 2 & 2 & 2 & 2 \\
\hline Post & M8 & 2 & 2 & 2 & 2 & 2 & 2 & 2 & 2 & 2 & 2 & 2 & 2 & 2 & 2 & 2 \\
\hline Pre & $\mathrm{H} 11$ & 2 & 2 & 2 & 2 & 2 & 2 & 2 & 2 & 2 & 2 & 2 & 2 & 2 & 2 & 2 \\
\hline Post & $\mathrm{H} 11$ & 2 & 2 & 2 & 2 & 2 & 2 & 2 & 2 & 2 & 2 & 2 & 2 & 1 & 1 & 1 \\
\hline
\end{tabular}


La respuesta predominante para la preevaluación fue 2, que implica no aceptar las situaciones descritas en los reactivos. En el postest, los datos no muestran cambios importantes. Se observa que la participante M8 no modificó sus respuestas tras haber asistido al taller. El participante H11 solo modificó tres de sus respuestas, en tanto la participante M1 modificó siete de sus respuestas que corresponden a los constructos 1 (depender del otro y adaptarse a él, postergando lo propio) y 3 (sentir que nada vale más que esa relación).

Se realizó un análisis detallado de los argumentos que las y los participantes daban a cada uno de los reactivos del cuestionario con el propósito de poder hacer una categorización de los argumentos presentados. Se generaron catorce categorías, y las que tuvieron más frecuencia (que fueron tres) se eligieron para presentarse en este reporte. A continuación, se describen cada una de estas categorías, y algunos ejemplos de los argumentos dados por los participantes:

Dependencia: Se refieren a argumentos que consideran inadecuados aquellos comportamientos destinados a sacrificar las actividades individuales con el fin de dedicar más tiempo y/o complacer a la pareja sentimental, renunciando a las propias responsabilidades y alejándose del resto de sus relaciones sociales.

Ejemplos: "Porque no debes depender de tu pareja para hacer cosas". "Crear este tipo de dependencia no está bien".

"Pues es un caso de codependencia".

Individualidad: En esta categoría se incluyen argumentos de los participantes que hacen referencia a que las situaciones planteadas no son adecuadas, pues consideran que la toma de decisiones no se tiene que realizar solamente con base en la relación de pareja. Suponen que la decisión de actuar o pensar debe recaer en uno mismo, sin dejarse influenciar por la pareja, por lo tanto, no se debe depender de las personas para la toma de decisiones y hay que dar prioridad a diversas actividades 
de la vida cotidiana, sobre todo aquellas que implican un desarrollo personal, sin mezclarlas con las relaciones sentimentales.

Ejemplos: "Porque el tener pareja no hace que forzosamente tengas que realizar todas las actividades con él o ella, ya que se puede diferir en gustos y preferencias".

"No es necesario que ocurra esto, todos tienen actividades por realizar y no es que a todo tiempo estén a tu disposición".

"No es adecuado porque su pareja es la que debe decidir si usarlos o no, él no tiene que decidir".

Decisión propia: Se expresa una determinación de realizar ciertas conductas basadas en las creencias del amor romántico aun cuando se tengan repercusiones negativas conocidas por el individuo que sufre violencia. En este caso particular, los participantes del taller no creen que sean adecuadas las conductas planteadas si estuvieran en esa situación, pero consideran que está bien o no hay problema en llevar a cabo estos comportamientos si la persona en cuestión está satisfecha o plena con su decisión, a pesar de que pueden ser señales previas de dependencia o hasta de violencia en la pareja.

Ejemplos:

"Pues si Antonio considera más importante el estar con su novia que hacer su trabajo, no encuentro problema".

"Yo no lo haría porque priorizo mis intereses, pero a quien lo haga no le veo problema".

"Si la persona se siente cómoda con la situación, pues está bien".

De acuerdo con lo presentado en la tabla 2, la categoría que tuvo más presencia en las dos evaluaciones fue la denominada decisión propia, pero ello ocurre con más frecuencia en la evaluación del postest. Podría decirse que el cambio que propició el taller en dos de los tres participantes fue el argumentar que la aceptación de comportamientos ligados a los mitos del amor romántico propiciadores de la violencia en la pareja es una decisión propia. La participante M8 no cambió su tendencia en como argumentar, pues puede observarse que en las dos evaluaciones predominan argumentos catalogables como decisión propia. En tanto que en los otros dos participantes si se ven cambios. La participante M1, que inicialmente empleaba argumentos de tres 
tipos, en la segunda evaluación favoreció los de decisión propia. En el participante H11, la fuerte presencia de los argumentos de individualidad después del taller es sustituida por los de decisión propia.

Se puede observar que la categoría otras tiene frecuencias más elevadas, se sugiere analizar esas respuestas y ver si pueden crearse categorías aglutinantes. Tal sugerencia se deja para otro reporte ya que durante el análisis inicial, el que aquí se presenta, se consideró que esas respuestas eran muy disímiles entre si.

\section{Tabla 3}

Frecuencia de las categorías dependencia, individualidad y decisión propia durante el pre y post test para cada integrante

\begin{tabular}{cccccc}
\hline \multirow{2}{*}{ Evaluaciones } & Participantes & Categorias & & \\
& Dependencia & Individualidad & Decisión propia & Otras \\
\hline Pre & M1 & 3 & 3 & 3 & 6 \\
Post & M1 & 1 & 2 & 5 & 7 \\
Pre & M8 & 0 & 2 & 7 & 6 \\
Post & M8 & 0 & 2 & 6 & 8 \\
Pre & H11 & 0 & 5 & 2 & 8 \\
Post & $\mathrm{H} 11$ & 3 & 1 & 5 & 6 \\
\hline
\end{tabular}

Con la intención de identificar como estas categorías se distribuían en cada uno de los constructos trabajados, se elaboró la tabla 4 únicamente con los reactivos del postest. En ella hay un agrupamiento: las categorías dependencia e individual se consideraron una representación de rechazo a los mitos del amor romántico y a la violencia de pareja, debido a que en ambas categorías el denominador común es que el argumento presentado es que los comportamientos guiados por los mitos no son adecuados; y la categoría de decisión propia se interpreta como una forma de 
diferenciación entre el sujeto que realiza el comportamiento acorde al mito romántico y el sujeto que responde el cuestionario.

\section{Tabla 4}

Distribución de las frecuencias de las categorías en relación con los constructos valorados en el post test

\section{Dependencia Individualidad Decisión propia}

a) Depender del otro y adaptarse a él, postergando lo propio

3

0

4

2

b) Prestar atención y vigilar cualquier signo de altibajos en el amor del

otro.

c) Sentir que nada vale más que esa relación todo en nombre del amor

e) Pensar que es

0 imposible volver a amar

con la misma intensidad

SUBTOTAL

4

4

16

TOTAL 
Puede advertirse nuevamente el predominio de argumentos de decisión propia, principalmente, en tres de los cincos constructos (en el a, el c y el e).

\section{Discusión}

En apariencia, los resultados no muestran efectos del taller. Desde antes de la impartición del taller, se presentó el rechazo a las situaciones que concuerdan con los mitos románticos y conducen a la violencia de pareja planteadas en la evaluación. Pero no hay que olvidar que los datos reportados son sólo de tres participantes. Lo anterior dificulta tener una idea clara de cómo se pudieran modificar las ideas ante los comportamientos que reflejan los mitos de amor presentes en el estudiantado universitario promovidos por los medios de comunicación.

Sin embargo, las investigadoras consideran que pueden advertirse algunos cambios. Se especula que la sensibilización estaba en proceso y que hubiese tenido un resultado más notorio con un mayor número de sesiones. Aunque, hay que señalar que la revisión de diversos reportes de talleres de sensibilización pone de manifiesto que el número de sesiones no parece ser una variable de alta importancia. De acuerdo con Virgilí (2014), los escasos talleres reportados en su mayoría constan de entre cuatro y nueve sesiones, y ella considera que la duración debe estar regulada en función de los contenidos. Para esta investigadora, uno de los tres elementos fundamentales a tomar en cuenta y a analizar es el referente al "proceder metodológico para la implementación de experiencias de sensibilización [...] evaluando la efectividad de los programas como procesos de aprendizaje, tanto a lo largo del mismo como tiempo después de su consecución" (pág. 27). Con relación a esta consideración, uno de los aspectos que faltó en esta experiencia -y que podría haber dado luz sobre sus efectos- fue el realizar una evaluación de seguimiento.

Los registros realizados durante la aplicación del taller muestran que las y los participantes son capaces de percibir los contenidos de los medios de comunicación como un factor que podría 
impulsar comportamientos negativos entre las parejas e instigar incluso la presencia de conductas violentas. También expresaron rechazo a los modelos difundidos en los medios como el adaptarse al otro postergando lo propio, perdonar hechos violentos, entre otros; y refirieron sentir que son conductas humillantes, que denotan una agresión y que podrían propiciar actos violentos.

Si bien los resultados del cuestionario mostraron que las y los participantes no están de acuerdo con la mayoría de las situaciones planteadas desde el pretest (lo cual podría ser una respuesta influenciada por la deseabilidad social), al momento de justificar su respuesta en el postest, es decir después del taller, predomina de manera general el tipo de respuesta de decisión propia como una razón válida para que las personas protagonistas en el cuestionario llevaran a cabo conductas basadas en los mitos del amor romántico y que podrían derivar en violencia hacia la pareja. Se menciona lo anterior porque en la redacción de los reactivos del cuestionario no se involucra personalmente a quien lo contesta, pues quienes realizan los comportamientos ahí descritos son "otros" individuos, no los participantes del taller, de ahí que se considere que pudiese existir una diferenciación en sus respuestas. Las y los participantes escriben frases como: "Yo no lo haría porque priorizo mis intereses, pero a quien lo haga no le veo problema "; "si Mariana considera que puede vivir y se siente a gusto en esa relación no hay problema, desde mi punto de vista yo la dejaría".

Este tipo de respuestas sugieren que las y los participantes sospechan que el individuo que realiza la acción ha tenido una reflexión sobre la situación que vive y aun así decide vivir situaciones que conducen a la violencia de pareja. Por consiguiente, las investigadoras del presente reporte proponen que el proceso de sensibilización estaba sucediendo. Aparentemente, ese acercamiento se estaba dando con el tipo de respuestas que expresaron los participantes; pero sólo los involucraba a ellos, y faltaba que se dieran cuenta de que eso podría ocurrir a otras personas, o bien, que consideraran que la situación podría extenderse a la sociedad manteniendo desigualdades. 
En este último escenario, era esperada la respuesta de las y los participantes para suponer que ya habían tomado conciencia de los comportamientos fomentados por los medios de comunicación y que pueden favorecer la conducta violenta en las relaciones de pareja en una comunidad amplia.

Por otra parte, la presencia de estas respuestas denominadas como decisión propia pudiera explicarse en función delo ocurrido en las sesiones del taller. En ellas se solicitaba que las y los participantes expresaran vivencias de situaciones que pudieran ser violentas o que condujeran a la violencia, o bien, que opinaran sobre situaciones observadas y a lo largo de las sesiones se desarrolló la capacidad de empatía hacia las personas que se encuentran inmersas en situaciones de violencia y/o dependencia emocional. Los participantes mostraron respeto, una postura neutra y sin prejuicios ante las vivencias y experiencias que se compartían dentro del taller. Es posible que por ello al término del taller prevaleciera el tipo de respuesta decisión propia.

Dado que las y los participantes son alumnos de sexto semestre de psicología, es posible otra interpretación, ellos podrían tener presentes algunos principios éticos del psicólogo, al parecer los relativos al respeto. Según la Federación Nacional de Colegios, Sociedades y Asociaciones de Psicólogos de México, A. C. (FENAPSIME) (2018) un objetivo de la difusión del código ético del psicólogo en los individuos que se forman como profesionales de la psicología es promover una actitud responsable, respetuosa y comprometida frente a los usuarios de los servicios psicológicos. El principio de respeto y defensa de los derechos humanos se expresa en el código de la FENAPSIME de esta forma: "Los profesionales de la psicología deberán respetar y promover los derechos fundamentales, la dignidad y el valor de todas las personas y no participarán en prácticas discriminatorias. Respetarán el derecho de los individuos a la privacidad, confidencialidad, autodeterminación y autonomía”. Pareciera entonces que los y las participantes del taller estuvieran respetando la privacidad de los supuestos individuos del cuestionario al presentar argumentos 
catalogados como decisión propia. Sin embargo, esta suposición tendría que investigarse más a fondo en otros estudios.

Además, si estuvieran presentes los principios éticos, sería parcialmente, pues en las respuestas no se argumentan aspectos relativos a otros principios éticos del psicólogo. Por ejemplo, en el código de tal Federación se estipula en el artículo 19 (subrayado de las autoras del reporte): “Cuando se atienda a personas víctimas ... de cualquier tipo de violencia que ponga en riesgo la vida o integridad de los usuarios o de una tercera persona ... se informará al usuario acerca de las instituciones especializadas que le brinden atención integral"; en el artículo 40: "La orientación (psicológica) es definida como un conjunto de acciones encaminadas al esclarecimiento que

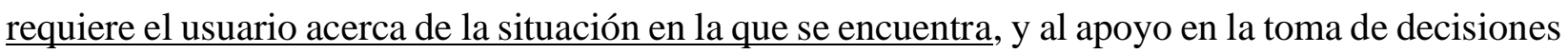
respecto al conocimiento de sí mismo y las relaciones que establece con su entorno las cuales se encuentran alterada"; y en el 41 “La consejería plantea un proceso de apoyo que trata de definir un estado emocional del usuario, y de hacerlo consciente de las situaciones que salen de su campo de percepción, al limitar su interacción efectiva con el contexto que lo rodea. El beneficio radica en que el usuario pueda obtener un cambio cognitivo y conductual ante problemáticas cotidianas. Esta forma de trabajo se distingue claramente de la Psicología clínica y de la psicoterapia”.

Si en las respuestas de los individuos que asistieron al taller se vieran reflejados argumentos que tomaran en cuenta estos aspectos, podría decirse que la respuesta catalogada como decisión propia sería una forma de responder de acuerdo con la formación identitaria de su profesión relativa al tema ético. Pero no se encontraron respuestas en esa categoría, ni en las otras que se generaron, que sugirieran que respondieron como psicólogos proporcionando, además de respeto, información, orientación o esclarecimiento a los supuestos sujetos de la evaluación. No obstante, se reitera que para fortalecer o rechazar esta suposición, es necesaria otra investigación en la que se tengan en cuenta estos aspectos, además de incluir más participantes. 
Desde otra perspectiva, se podría decir que estas respuestas que establecen una diferenciación entre una situación observada (la planteada en el instrumento) y una situación vivida personalmente pudieran en un momento dado ser interpretadas como una excusa del acto violento bajo el supuesto de que este es asumido por “decisión propia”. Pero ello no necesariamente es así. No es una excusa decir que el acto se realice, siempre y cuando se tenga claridad de las implicaciones que este conlleva y se debe dejar al individuo que lo realice. Quizás deba acentuarse que lo que hace falta saber es si el otro tiene claridad de esas implicaciones. Como eso es difícil de saber, probablemente hubiese sido necesario que en el taller se enfatizara no sólo el plano individual, sino también el colectivo, en donde se acentuara que como comunidad hay que prevenir y disminuir esos mensajes en los medios de comunicación, o bien, incrementar aquellos que den cuenta de las implicaciones de los comportamientos asociados a los mitos del amor romántico que conducen a la violencia para que la población en general no los siga manifestando.

Por otra parte, el registro de lo expresado por los participantes durante algunas sesiones del taller respalda que en varias ocasiones comentaron que no se habían percatado de las implicaciones violentas de los mensajes que eran presentados en los diversos medios de comunicación y que ello hacía que el taller fuese valioso, pues les permitió reflexionar sobre las repercusiones de esos mensajes en los comportamientos amorosos y que pueden conducir a la agresión en las relaciones de pareja.

Se propone para futuros talleres, promover la reflexión de que, como comunidad, se deben favorecer comportamientos alternativos a los fomentados por los medios de comunicación y derivados del amor romántico que conducen a la violencia de pareja. Con ello, se estaría cumpliendo con la propuesta de Virgilí (2014) de intervenir en los imaginarios instituidos para impulsar procesos de transformación potenciando contenidos emergentes (imaginarios instituyentes) en un contexto colectivo. Dicho en otras palabras, en el futuro taller se propone dar 
crédito a las propuestas alternativas que proporcionen las personas asistentes, explicitarlas, fortalecerlas, legitimarlas y ponerlas en el centro de la reflexión grupal. De esa forma, se espera que las significaciones e imaginarios instituidos (las impuestas por la sociedad y los medios de comunicación) se deconstruyan y den lugar a los instituyentes.

\section{Conclusiones}

De manera general, conforme al análisis realizado se puede inferir que el taller transformó, parcialmente, los modelos y prácticas de los y las participantes. Estos ya contaban con información sobre violencia, pero en el taller reconocieron y reflexionaron sobre el impacto de los mitos del amor romántico en una relación de pareja y su relación con la violencia. Como resultado de ello, sus respuestas ante situaciones que ejemplificaban algunos comportamientos derivados de esos mitos mostraron argumentos catalogados en este trabajo como decisión propia, dejando con ello de manifiesto que hacían una diferenciación entre ellos y otros individuos y que el proceso de sensibilización se había iniciado, aunque faltaba que dieran respuestas como integrantes de una comunidad.

Se considera de suma importancia que en las próximas investigaciones o propuestas de taller que aborden el tema de los mitos del amor romántico asociados con la violencia en la pareja se adopte una perspectiva donde se cuestionen los productos culturales que mantienen dichos mitos. También, se sugiere que se ahonden en las situaciones y argumentaciones descritas por las y los participantes que se asemejen a la categoría decisión propia definida en este trabajo, ya que puede ser una creencia arraigada que permite minimizar y hasta invisibilizar los diferentes casos y tipos de violencia de pareja; así pues, se propone se tomen como un punto de partida para el inicio de un proceso de reflexión y sobre todo de reconstrucción de los mitos del amor romántico que permita a los individuos y su comunidad disminuir la violencia de pareja asociada a ellos. 


\section{Referencias}

Cabero, J. y Romero, R. (2001). Violencia, juventud y medios de comunicación. Comunicar. Revista Científica de Comunicación y Educación, 17, pp.126-132. https://www.redalyc.org/pdf/158/15801719.pdf

Castro, R. y Casique, I. (2010). Violencia en el noviazgo entre los jóvenes mexicanos. México: Centro Regional de Investigaciones Multidisciplinarias, UNAM. https://www.crim.unam.mx/web/sites/default/files/Violencia\%20en\%20el\%20nov iazgo.pdf

CONAPO (2014). Las relaciones de pareja, un enfoque estadístico. [Comunicado de prensa]. Consultado el 18 de marzo de 2020 http://www.conapo.gob.mx/es/CONAPO/13_de_febrero_Informacion_estadistica _sobre_las_relaciones_de_pareja

Deza, S. (2012) ¿Por qué las mujeres permanecen en las relaciones de violencia? Avances de Psicología, $\quad 20 \quad$ (1), $\quad$ pp. http://www.unife.edu.pe/publicaciones/revistas/psicologia/2012/sabinadeza.pdf

FENAPSIME (2018). Código de Ética de las y los Psicólogos Mexicanos. México: Federación Nacional de Colegios, Sociedades y Asociaciones de Psicólogos de México, A. C. https://fenapsime.org/2018/01/01/codigo-de-etica-de-las-y-lospsicologos-mexicanos/

Ferrer, V. y Bosch, E. (2013). Del amor romántico a la violencia de género. Para una coeducación emocional en la agenda educativa. Revista de Currículum y Formación $\begin{array}{lllll}\text { de } & \text { Profesorado, } & 17, & (1), & 105-\end{array}$ https://unidaddegenerosgg.edomex.gob.mx/sites/unidaddegenerosgg.edomex.gob. 
mx/files/files/biblioteca/1\%20TEORI\%CC\%81A\%20DE\%20GE\%CC\%81NERO/ Amor\%20roma\%CC\%81ntico\%20y\%20violencia\%20de\%20ge\%CC\%81nero.pdf García-Carpintero, M. Rodríguez-Santero, J. y Porcel-Gálvez, A. (2017). Diseño y validación de la escala para la detección de violencia en el noviazgo en jóvenes en la Universidad de Sevilla. Gaceta Sanitaria, 32, (2), 121-128. https://www.scielosp.org/pdf/gs/2018.v32n2/121-128

Galicia, I. X., Robles, F. J. y Sánchez, A. (2020). La violencia psicológica de pareja en el estudiantado universitario como una manifestación de la violencia escolar en la FES Iztacala. En A. L. Robles (coord.) Diagnóstico Institucional sobre Violencia Escolar en la Facultad de Estudios Superiores Iztacala. México: FES Iztacala. UNAM.

Galicia, I. X., Robles, F. J., Sánchez, A. y Núñez, E. (2019). Dependencia emocional y mitos del amor en estudiantes de dos niveles educativos. Revista Iberoamericana $\begin{array}{llll}\text { de } & \text { Psicología, } & 12 & \text { (3), }\end{array}$ https://reviberopsicologia.ibero.edu.co/article/view/1637

Linares, C. L. y Sologuren, G. N. (2012). Significados de las relaciones de pareja en las y los estudiantes universitarios/as y sus implicancias en sus interacciones sociales de la Universidad Nacional Jorge Basadre Grohmann Tacna, 2011. Ciencia y Desarrollo, 14 , 78-84. http://revistas.unjbg.edu.pe/index.php/CYD/article/view/307

Luzón, J. M., Recio, P., Ramos. E. y Peña, E. (2011) Factores de Riesgo y de Protección en la Prevención contra la Violencia de Género en la Pareja. Andalucía: Instituto Andaluz de la Mujer. Consejería para la Igualdad y Bienestar Social. www.fundacionmujeres.es/img/Document/15656/documento.pdf 
Montaño, L. (2015). Representación y violencia simbólica. Una reflexión acerca de la modernización de las universidades públicas en México. En G, Sánchez e I. Sánchez. (Coord.) Miradas Críticas a la Complejidad de la Violencia Universitaria. México: Fontamara.

Nadal, A. (2002). De los celos al amor imposible (o de Otelo al mito del andrógino) XIV Congrés Valenciá de Filosofía, Valencia: Societat de Filosofia del País Valencià, 139-154. https://www.uv.es/sfpv/congressos_textos/congres14.pdf

Olvera, J., Arias, J. y Amador, R. (2012). Tipos de violencia en el noviazgo: Estudiantes universitarias de la UAEM, Zumpango. Revista Electrónica de Psicología Iztacala, 15(1),

pp.

$150-171$. http://www.revistas.unam.mx/index.php/repi/article/view/30908

Pascual, A. (2016). Sobre el mito del amor romántico. Amores cinematográficos y educación. Revista de Educación y Humanidades, 10, pp. 63-78. https://revistaseug.ugr.es/index.php/dedica/article/view/6850/6623

Rivera-Rivera, L., Allen, B., Rodríguez-Ortega, G., Chávez-Ayala, R. y Lazcano-Ponce, E. (2006). Violencia durante el noviazgo, depresión y conductas de riesgo en estudiantes femeninas (12-24 años). Salud Pública de México, 48, 288-296. http://www.scielo.org.mx/pdf/spm/v48s2/31385.pdf

Rodríguez-Franco, L., López-Cepero, J., Rodríguez, F. J., Bringas, C., Antuña, M. A. y Estrada, C. (2010). Validación del cuestionario de violencia entre novios (CUVINO) en jóvenes hispanohablantes: Análisis de resultados en España, México y Argentina. Anuario de Psicología Clínica y de la Salud, 6, 45-52. http://institucional.us.es/apcs/doc/APCS_6_esp_45-52.pdf 
Rojas, S, J. (2013). Violencia en el noviazgo de adolescentes mexicanos: Una revisión. Revista de Educación y Desarrollo, 27, pp. 49-58. https://doi.org/10.33670/18181023.v12i02.71

Rosales, M., Flórez, M. Madan, L. y Fernández, T. (2017). La violencia de pareja: análisis en una población universitaria de Santo Domingo. Enseñanza e Investigación en $\begin{array}{lll}\text { Psicología, } & \text { 22, } & \text { (2), }\end{array}$ https://www.academia.edu/33664650/La_violencia_de_pareja_análisis_en_una_p oblación_universitaria_de_Santo_Domingo_2017_

Samaniego, E. y Freixas, A. (2010). Estudio sobre la identificación y vivencia de violencia en parejas adolescentes. Apuntes de Psicología, 28 (3), pp. 249-366. http://www.apuntesdepsicologia.es/index.php/revista/article/view/224

Sánchez, A. R. y Solís, M. J. (2009). Las huellas de la violencia en el noviazgo: estudiantes universitarios y trayectorias escolares de la FES Acatlán. En XI Congreso Nacional de Investigación Educativa, 21-25 de septiembre de 2009. Veracruz: COMIE. http://www.comie.org.mx/congreso/memoriaelectronica/v10/pdf/area_tematica_16 /ponencias/0780-F.pdf

Virgilí, D. (2014). Reflexiones teórico-metodológicas sobre sensibilización y capacitación en género. Apuntes de una propuesta para su implementación en contextos grupales. La Ventana, Revista de Estudios de Género, 40, pp. 7-58. http://www.scielo.org.mx/scielo.php?script=sci_arttext\&pid=S140594362014000200003

Zamudio, F. J., Andrade, M. A., Arana, R. I. y Alvarado, A (2017). Violencia de género sobre estudiantes universitarios(as). Convergencia. Revista de Ciencias Sociales. 
75, 133. http://www.scielo.org.mx/scielo.php?script=sci_abstract\&pid=S1405$14352017000300133 \& \operatorname{lng}=$ es\&nrm=iso

Cómo citar este artículo: Chora López , M. A., Galicia Moyeda, I. X., \& Guerrero García , B. G. . (2021). Taller de sensibilización ante la violencia de pareja promovida por los mitos del amor romántico. Psicumex, 11, 1-29, e369. https://doi.org/10.36793/psicumex.v11i2.369 